\title{
Procollagen Type I N-Terminal Peptide
}

National Cancer Institute

\section{Source}

National Cancer Institute. Procollagen Type I N-Terminal Peptide. NCI Thesaurus. Code C116963.

A polypeptide comprised of the amino-terminal portions of procollagen, which are proteolytically removed during the intracellular formation of type I collagen fibrils. Increased plasma or serum concentrations of these peptides are associated with both fibrillogenesis of type I collagen and bone formation. 